\title{
A CAD Based Concept Car Workflow
}

\author{
Allan D. Spence, Ph.D., P.Eng. \\ Associate Professor, Mechanical Engineering \\ McMaster University \\ adspence@mcmaster.ca
}

\begin{abstract}
This paper describes a Computer Aided Design (CAD) based workflow for constructing some basic elements of a concept automobile. Students begin by choosing existing or new concept vehicle wheel base, track width, etc. dimensions. These are then laid out to 1/15 scale in 2-D, and used to determine steering geometry. A CAD anthropometric model is used to guide interior seating, and instrument panel design. Instead of a differential, a one rear wheel drive mechanism is used. The body shell is created using surface modeling and rapid prototyping. The flat plastic sheet chassis base holes and body shell profile are machined. The remote control steering servo and wheel drive components are purchased.
\end{abstract}

\section{Introduction}

A goal of the McMaster Faculty of Engineering Strategic Plan is to offer interdisciplinary undergraduate design courses [1]. This paper describes initial implementation of a scale model concept car creation project included as part of the McMaster University Mech Eng 4Z03/6Z03 Computer Aided Design / Manufacturing / Engineering (CAD/CAM/CAE) course offered jointly to both senior year Mechanical Engineering and Mechatronic Engineering students, and as a transitional Master's graduate level course.

The remainder of the paper is organized as follows. In Section 2, the project parameters are introduced, including 2-D chassis base layout, steering mechanism requirements, interior cabin design of seats and instrument panel, and outer body shell. Conversion of parameters to design expectations is covered in Section 3. Section 4 describes the CAD implementation workflow in detail. Section 5 reflects on initial observations and future implementation plans. The paper is summarized in Section 6 .

\section{Project Parameters}

Each student was required to choose either an existing automobile to model, or, with approval, a new concept car. Essential parameters such as the wheel base and track width, two or four door, etc. represented the first reportable milestone. For existing vehicles, brochure or equivalent photograph images were used to establish a sense of scale (Fig. 1(a)). For concept cars, students were encouraged to adapt an existing design using photo image editing software.

Simple Darwin-Ackermann Steering [2] (Fig. 1(b)), specific to the chosen wheel base and track parameters, was required. A maximum steering angle of 30 degrees was required. Standard fischertechnik [3] components and corresponding CAD models for tires, rims, servos, rear wheel drive gearing, motors, infrared remote control receivers, and battery holders, were provided to the students.

The interior cabin was to have sufficient leg room, head room, and seating width. To assist with a sense of scale, the iMike [4] (Fig. 1(c)) anthropometric CAD model was provided, plus reference to standard ergonomic data [5].

Where possible, students were expected to use standard fasteners (nuts, screws, washers, or fischertechnik parts). The body shell had to include curvature continuous wheel well designs, and a cabin roof that provided adequate head room. Bosses had to mate with corresponding sockets on chassis base components. The body shell was to be rapid prototyped, along with any other small functional components. Mounting holes in the chassis base were drilled, and the outside profile was to be CNC machined to match the body shell.

Autodesk [6] Inventor was used for general CAD design, and Alias was used for the body shell design. EdgeCAM [7] was used for chassis base CNC machining, and a Dimension BST [8] rapid 


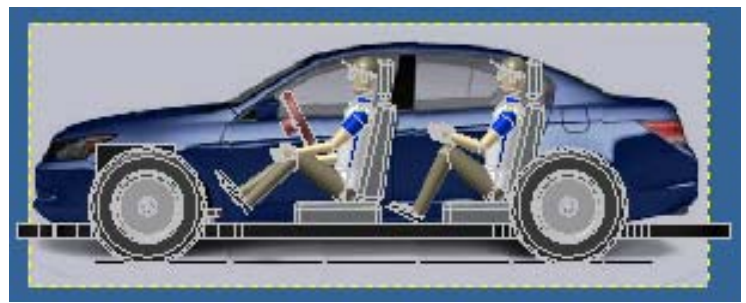

(a)

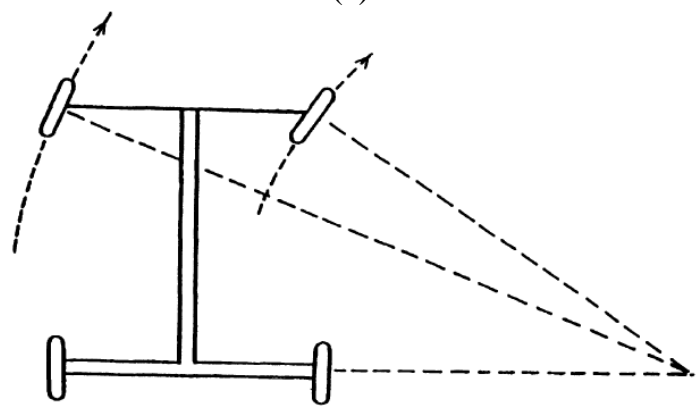

(b)

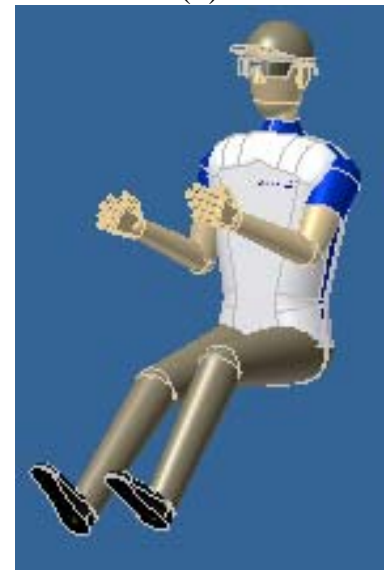

(c)

Figure 1. (a) vehicle photograph image used for scaling; (b) Darwin-Ackermann steering geometry [2]. The front wheel axes should intersect at a common point with the rear axle; (c) iMike [4].

prototyping machine was used for small mating parts and the body shell.

\section{Design Expectations}

\subsection{Chassis Base Layout}

Suspension was not considered. This simplification reduces the steering geometry to a trapezoid, with the knuckle angles (passing through the kingpin) chosen to intersect at the centre of the rear axle (Fig. 2). A fischertechnik servo was used to drive the tie rod, with a slot used to provide the necessary degree of freedom. Because it has little influence over practical values [9], effect of the knuckle length was not specifically

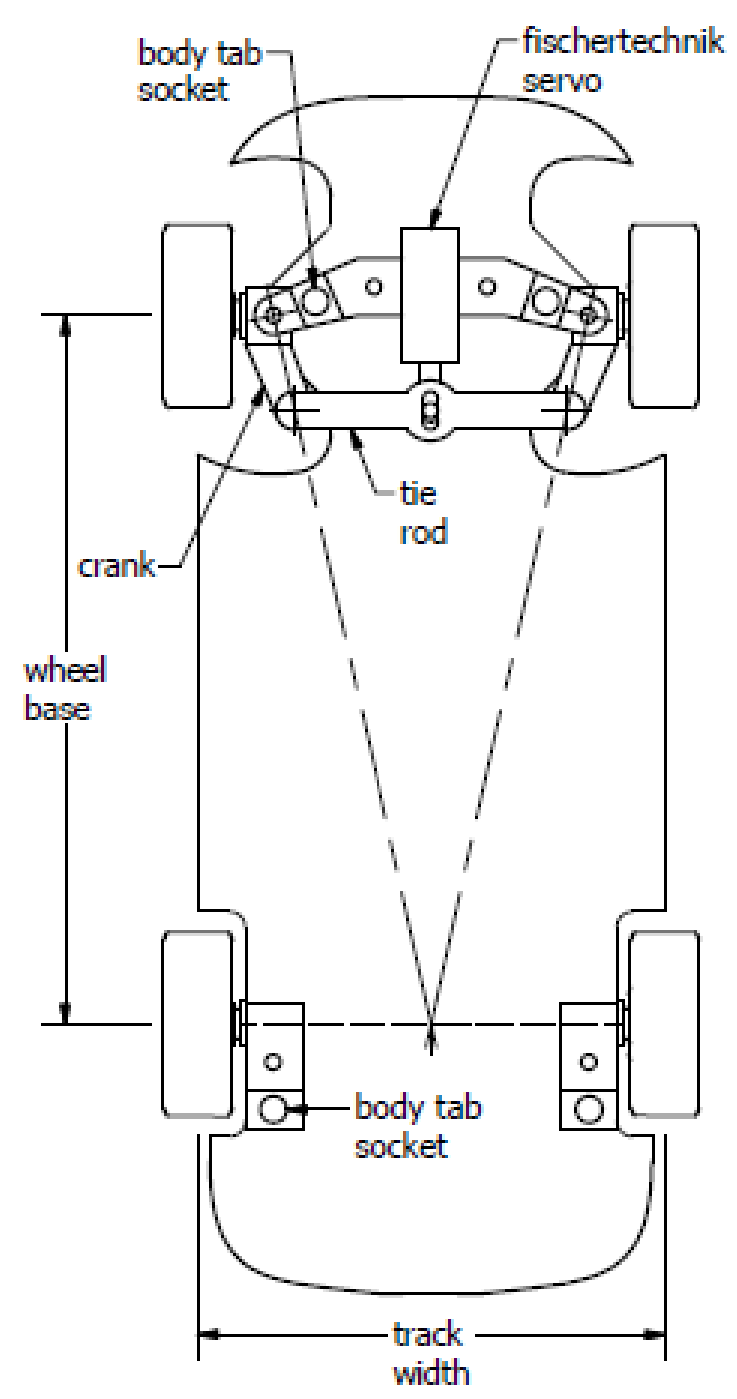

Figure 2. Chassis base layout

addressed. The single wheel drive was applied to the left rear wheel. Metric M4 fasteners were added to secure the components, and, after the body shell design was completed, the surface was used to trim the outer chassis base profile within the CAD model. This profile was used to generate a CNC program. The chassis base fastener holes were drilled, and then used to secure the frame to a machining fixture. The profile was then cut using a small hobby sized machine tool.

\subsection{Interior Cabin}

Because of the small scale, seats were designed as a single part that could be attached through the chassis base using a single fastener. Again, the reference vehicle photograph was used to provide guidance on seat positioning. The steering wheel included finger 


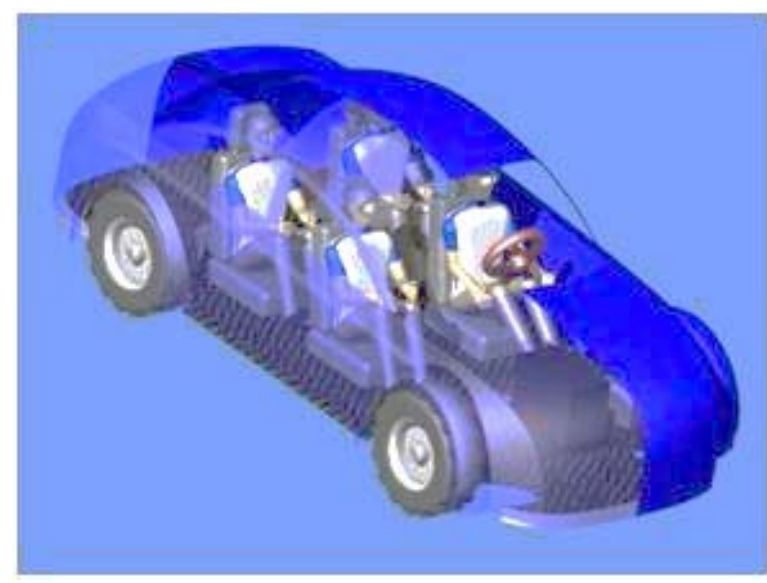

Figure 3. Scale model in CAD. The instrument panel is omitted for clarity.

grips and a center hole for an M4 fastener to attach it to the instrument panel.

\subsection{Body Shell}

The body shell design procedure was based on the workflow described in [10]. The partially assembled model was imported into Alias to ensure head room clearance, match of wheel well positions, etc. Tabs were added to mate with the chassis base components. For learning use and to save material, only one side of the body shell was rapid prototyped.

The instrument panel was attached to the chassis base using a centre console and fasteners. Clearance had to be included for the front wheel wells and steering tie rod. A good fit with the body shell front window opening was also expected. These parts were all rapid prototyped, and then assembled with the chassis base components. An example of a completed CAD model is shown in Fig. 3. The actual physical model is shown in Fig. 4.

\section{CAD Implementation Workflow}

For teaching Computer Aided Design, this project represents a paradigm shift from "just in case" subject based teaching to "just in time" learning of skills and theory required to complete the workflow. It is consistent with the Conceive-Design-ImplementOperate (CDIO) [11] syllabus, and life-long learning.

\subsection{Workflow and Milestone Requirements}

Students require clear guidelines and workflow milestones. This is a particular issue with design projects, where the "fuzzy front end" [12] often leads to procrastination. Posing the project as a scale model of a full size automobile came across as artificial rather than serious. Student motivation increased when it was recast as a legitimate toy product that
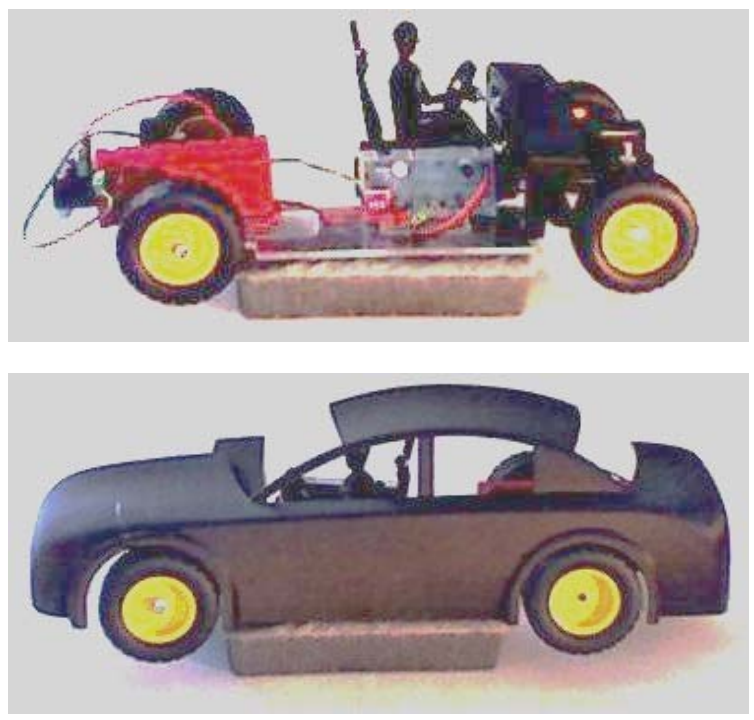

Figure 4. Completed scale model as built.

could actually be manufactured and sold. This allowed reference to the design workflow chapter divisions, including concept generation and selection, used in the widely adopted Ulrich and Eppinger textbook [12] that many students were familiar with from a prior course. Mandatory use of fischertechnik components and standard fasteners also reduced concept choice indecision.

\subsection{The Value of Sketching}

Particularly in a Computer Aided Design course, students tend to leap into using the 3-D solid modeler software without any preparation, although the value of concept sketches remains acknowledged [14]. Our simple conclusion is that we need to provide formal instruction in concept sketching, and for next year this will be approached using two Wacom [15] tablets. Video lessons will be prepared by knowledgeable School of the Arts colleagues and an Industrial Design sessional instructor. The tablets will be available for students to use in the CAD laboratory, and submission of sketches will be a graded milestone. This might be a high technology approach to bring attention to sketching, but it is hoped that renewed sketching skills will be retained and transferred to pencil and paper.

\subsection{Plotting to Scale in 2-D}

Initial 2-D planar layout of the wheel bearing blocks, steering components, seating, and drive components provides a useful next milestone. The fasteners are provided as a standard component library with the CAD system, and fischertechnik CAD models were also provided to the students. At this point a 1:1 scale plotting confirms that the layout is workable, and an $\mathrm{XY}$ hole coordinates table documents information required for chassis base drilling using a small manual 


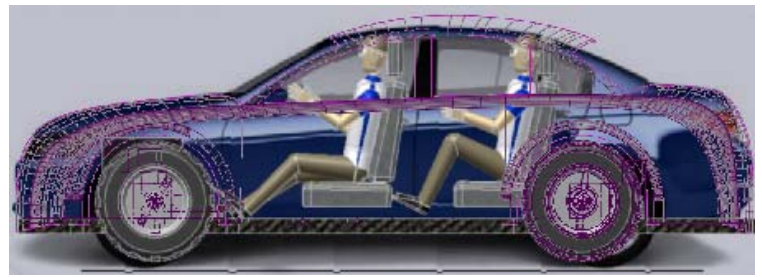

Figure 5. Alias surface model

Digital Read Out (DRO) machine tool. Any custom steering parts are rapid prototyped and students assemble the components as a major midterm submission for grading. Tangible assembly additionally provides motivation for the remaining project steps.

\subsection{Interior Cabin Seating}

The interior cabin and body shell design steps in the project are geometrically much more difficult to teach without resorting to detailed B-spline [16] mathematics explanations. For the initial implementation, students were provided with the iMike CAD model and were required to design seat and backrest/headrest lofted surfaces from B-spline curves. Online video lessons were provided to assist. At the small scale, features were not clearly visible, and results were not convincing. Because it was used for the chassis base layout, Inventor was chosen for this task. This was a mistake as Inventor is not well equipped for surface modeling. For the next offering, the intention is to provide pre-made iMike / seat CAD models and rapid prototyped parts.

\subsection{Exterior Body Shell}

More success was observed with the body shell design using the Autodesk Alias surface modeler. This is attributed to the fact that Alias is a much superior tool for this task, and the excellent textbook instructions. Students would nonetheless encounter difficulties with continuity between surfaces, and this provided an opportunity for the instructor to offer mathematical explanations to reinforce B-spline theory understanding.

To draw the body shell, the chassis base, seats, iMike, wheels, steering and drive components were first imported from Inventor to Alias. Side / top / front photos of the intended car were added as image canvasses (Fig. 5). Sketches could be used for concept cars where no photo was available. The completed body shell was exported back to Inventor as a zero thickness surface to verify correct fit, and to trim the chassis base outer profile for CNC machining (Fig. 6). Students were very motivated and several excellent body shell designs were created (Fig. 7). For this year, the body shells were not actually produced for the lecture course. Representative manufactured student

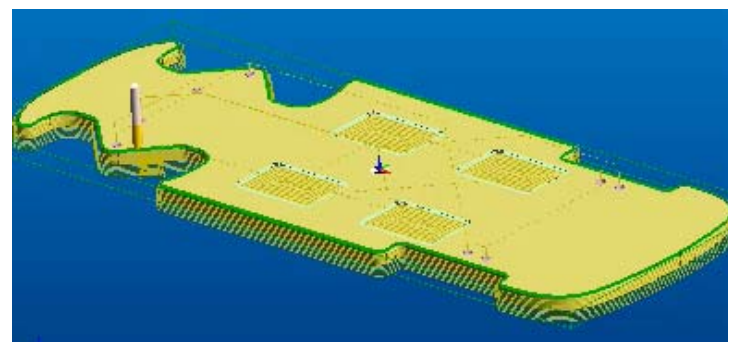

Figure 6. Chassis base profile CNC tool path generation

team models from a capstone course are shown in Fig. 8.

\section{General Observations / Future Plans}

Digital prototyping is now standard in engineering product design, and Computer Aided Design systems are at the core. Efficacy in using such systems is expected by employers, and in response CAD courses have been introduced by most engineering schools. Being such a hands-on topic, regular lectures led by instructor provide little student motivation. Available textbooks are usually mathematics / computer science oriented - leading to further lack of engineering student enthusiasm. To address this, a concept car CAD project was introduced. Web based video lessons were recorded to avoid dependence on live lecture demonstrations.

For the first time, Mechatronics and Mechanical Engineering students were brought together in the same CAD course, and the car project, with electronic remote control, was chosen to appeal to both populations. The project was well received by students, and with the observations and planned improvements noted earlier, it is expected to remain popular.

As always, there is pressure to include more in a four year engineering curriculum, perhaps to the point of overwhelming students. Some would suggest that students should simply learn CAD on their own. Recent internal survey responses from our students, however, indicate that they very much expect some training in how to use computer design and other engineering communication tools. External program reviewers have agreed. The developed project does this in a more "just-in-time" manner than usual lecture course formats, and this makes better use of limited student time.

For many years, the CAD course was offered as a one evening per week elective. Students on work terms would then sign up for the course to reduce their load after they returned to campus for a regular academic term. This suggests that an online course offering format might be attractive, or a concentrated 

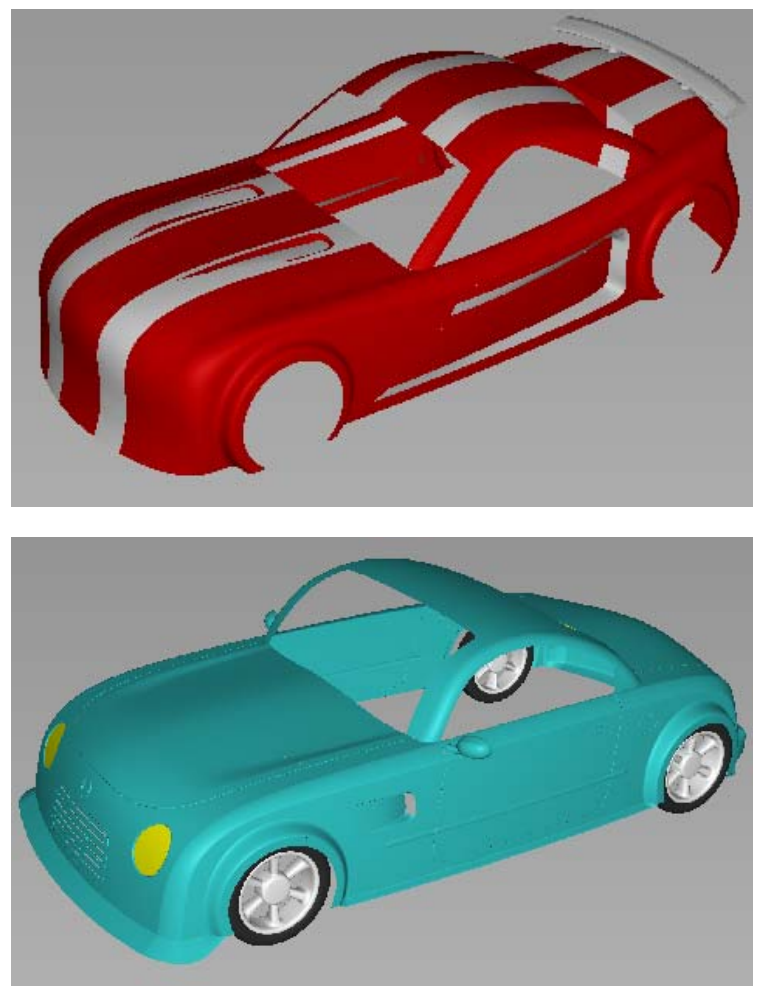

Figure 7. Student design examples

module format such as the Thursday-Sunday method used by the ADMI Industrial Master's program [17]. The student software licensing rules offered by the vendors, combined with ever increasing internet speeds, make this an option of interest. Some institutions use leased laptop computer programs, but we currently are more inclined towards student owned computers with a special "technical setup" day occurring at the beginning of the course. Thin client computing is also growing, and has already been adopted on campus for use in our Year 1 CAD laboratory. Interest in collaboration and resource sharing with other universities is welcome, and can provide an opportunity to more quickly accumulate systematic student efficacy knowledge.

It is recognized that a particular design project can only be used for a few years before issues of academic honesty, and software obsolescence, arise. On the other hand, it is difficult for the instructor to broadly change parameters every year. The intention is to maintain the concept car idea for the immediate future. For example, next year, the specification will be for a two door urban eco-concept car [18] with a much shorter wheelbase. Increased emphasis on sketching, regular milestone, and individual submissions, is expected to maintain original effort. By remaining with a product rather than CAD software focus, dependence on a particular vendor or release is minimized.
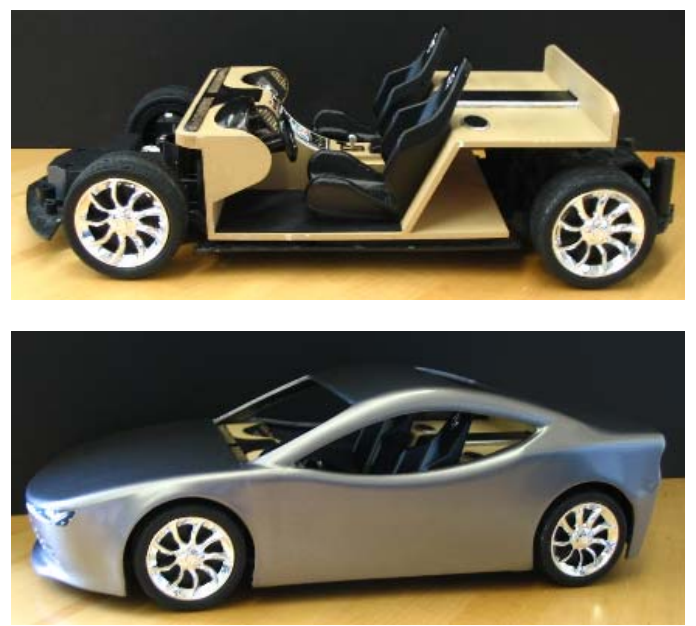

Figure 8. Capstone course concept car model

Costs for components and rapid prototyping are also a concern. Indications are that students are prepared to purchase items for a tangible project that they can keep as an addition to their portfolio, to show potential employers, etc. Ordering these for each student is currently being arranged with the fischertechnik vendor, and each student will be expected to purchase the items needed for the 2-D chassis base and steering milestone implementation, at a cost of approximately $\$ 60$. Short three month terms make it difficult to promptly rapid prototype numerous large parts. Body shell academic requirements will therefore stop at CAD documentation. An additional high resolution rapid prototyping machine will be available for next year, and students wishing to produce their body shell can purchase the plastic (estimated cost \$80) and arrange for it to be produced after the end of lectures (during examinations).

\section{Conclusions}

Engineering students required Computer Aided Design skills, but are not motivated by currently available curriculum and lecture style teaching. As an alternative, a Computer Aided Design (CAD) based workflow for constructing some basic elements of a concept automobile is described in this paper. The project is presented as a $1 / 15$ scale model that is to be documented, actually made and taken home. Reception of the project was positive, and curriculum improvements to address observed problem areas is ongoing. Ultimately it is anticipated that the course lectures will be replaced by web based notes and video lessons, with the instructor then redirecting attention to laboratory interaction with the students. Interest in collaboration from other institutions is invited. 


\section{Acknowledgements}

The author extends his appreciation to the students in Mech Eng 4Z03 for their co-operation, to Ian Mackenzie and Y.A. (Allen) Liu for their Teaching Assistant help, and to Anatoly Dolgopolov for development and documentation assistance in preparation for the 2011 course offering. Studica, Inc. (Derek Murphy) donated the fischertechnik components and one Wacom tablet. The second Wacom tablet, and new Hewlett-Packard computers for the CAD laboratory, were granted through the Partners for the Advancement of Collaborative Engineering Education [19]. Funding for Anatoly's position was provided through a McMaster University Centre for Leadership in Learning Imperial Oil Charitable Foundation Departmental Teaching Innovation Award received from an application jointly prepared by the author, Dr. Robert Fleisig, and other members of the McMaster Mechanical Engineering Design Curriculum group.

\section{References}

[1] Faculty of Engineering, Engineering a Sustainable Society, Strategic Plan 2009-2014, McMaster

University, 2009.

www.eng.mcmaster.ca/strategicplan/StrategicPlan.pdf

[2] D. King-Hele, “Erasmus Darwin’s Improved Design for Steering Carriages - and Cars”, Notes Rec. R. Soc. Lond., 56(1), 41-62, 2002.

[3] fischertechnik GmbH, Waldachtal, Germany, www.fishertechnik.de.

[4] M.V. Ficarra, iMike, Cross Bros. Co. Inc., Rochester, NY.

http://inventorparts.com/Parts/Ergonomics/imike.zip.

[5] M. Helander, A Guide to Human Factors and Ergonomics, 2nd Ed., CRC Press, 2006.

[6] Autodesk, Inc., San Rafael, CA, www.autodesk.com.

[7] Planit-EdgeCAM, Reading, Berkshire, UK, www.edgecam.com
[8] Stratasys, Inc., Dimension BST, Eden Prairie, MN, USA, www.dimensionprinting.com.

[9] P.A. Simionescu and D. Beale, "Optimum Synthesis of the Four-Bar Function Generator in its Symmetric Embodiment: The Ackermann Steering Linkage”, Mechanism and Machine Theory, 37, 14871504, 2002.

[10] Alias Systems Corp., Learning Design with Alias StudioTools, Sybex (Wiley), 2005.

[11] E. Crawley, J. Malmqvist, S. Ostlund, and D. Brodeur, Rethinking Engineering Education; The CDIO Approach, Springer, 2007.

[12] P.G. Smith and D.G. Reinertsen, Developing Products in Half the Time: New Rules, New Tools, 2nd Ed., Wiley, 1997.

[13] K. Ulrich and S. Eppinger, Product Design and Development, 4th Ed., McGraw-Hill, 2007

[14] D. McCormick, "Seeing Mechanical: A Case for Advancing the Role of Sketching in the Art of Engineering”, Mechanical Engineering, ASME, Sep. 2007.

[15] Wacom Co. Ltd., Saitama, Japan, www.wacom.com.

[16] L.A. Peigl and W. Tiller, The NURBS Book, 2nd Ed., Springer, 1996.

[17] Advanced Design and Manufacturing Institute, www.admicanada.com

[18] Shell Oil Company, Houston, TX, “A Facelift for Shell's Eco-marathon Urban Concept Car”, Press Release Mar. 29, 2010, http://www.shell.com/home/content/ecomarathon/ame ricas/media/2010_sema_houston_mayor.html

[19] Partners for the Advancement of Collaborative Engineering Education, www.pacepartners.org 